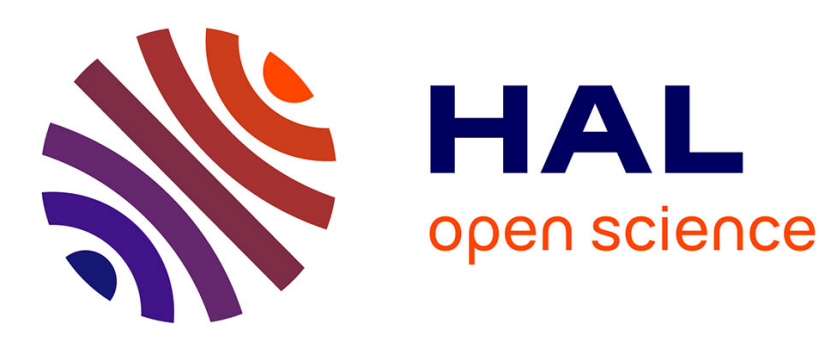

\title{
Inelastic scattering of a pulsed electron beam from a molecular wave packet
}

\author{
M. Ferrero, F. Robicheaux
}

\section{To cite this version:}

M. Ferrero, F. Robicheaux. Inelastic scattering of a pulsed electron beam from a molecular wave packet. Chemical Physics, 2001, 267 (1-3), pp.93-98. 10.1016/S0301-0104(01)00265-8 . hal-02364173

\section{HAL Id: hal-02364173 \\ https://hal.science/hal-02364173}

Submitted on 19 Nov 2020

HAL is a multi-disciplinary open access archive for the deposit and dissemination of scientific research documents, whether they are published or not. The documents may come from teaching and research institutions in France or abroad, or from public or private research centers.
L'archive ouverte pluridisciplinaire HAL, est destinée au dépôt et à la diffusion de documents scientifiques de niveau recherche, publiés ou non, émanant des établissements d'enseignement et de recherche français ou étrangers, des laboratoires publics ou privés. 


\title{
Inelastic scattering of a pulsed electron beam from a molecular wave packet
}

\author{
M. Ferrero and F. Robicheaux ${ }^{1}$ \\ ${ }^{1}$ Department of Physics, Auburn University, Auburn, AL 36849
}

(Dated: November 19, 2020)

\begin{abstract}
We present a theory for the scattering of a short electron pulse from a molecular wave packet. We focus on the transition between two electronic states and show how transition probabilities as a function of internal nuclear positions can be obtained. Also, a pulsed electron beam can be used to control the transition probabilities to different electronic levels. We demonstrate the validity of the theory by comparing to a direct numerical solution of Schrödinger's equation in a model system.
\end{abstract}

Recently, we have begun investigating ${ }^{1,2}$ the new phenomena that can be observed if scattering theory is generalized to describe the interaction of a quantum target with a matter beam that varies on time scales as fast or faster than the target. Although no experimental investigations with rapidly time varying matter beams have been performed to date, such beams have been created for electrons ${ }^{3,4}$ and for slow atoms using atom lasers ${ }^{5-8}$. Soon, it may be possible to use pulsed matter beams in to cause inelastic transitions in practical experiments, thus it is timely to begin theoretical investigations into specific arrangements. In this paper, we show how the combination of a pulsed electron beam and a molecular wave packet can be used to directly determine the dependence of an electronic transition probability on the nuclear positions. We also discuss how a pulsed electron beam in combination with a molecular wave packet can be used to control the scattering transition to particular final states.

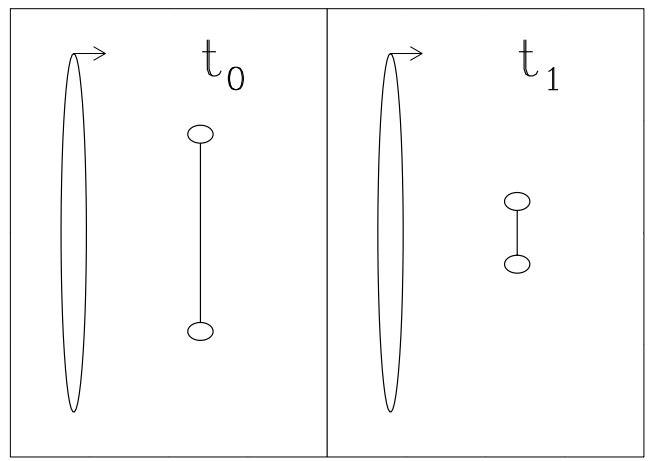

FIG. 1. A schematic drawing of a pulsed electron beam scattering from a molecular wave packet. At $t_{0}$ the nuclear wave packet is localized at the outer turning point; at $t_{1}$, the nuclear wave packet is localized at the inner turning point. In both frames, a circular sheet of electron density is incident on the molecule. If the duration of the interaction of the electron sheet with the molecule is short compared to the rovibrational periods, the nuclei are essentially frozen during the whole of the interaction.

The type of system that we are investigating is sketched in Fig. 1. A molecule is prepared in a wave packet of the nuclear positions; this packet could be in the inter-nuclear distances or in the orientation of the molecule. A single-pulse electron beam interacts with the molecule and causes an electronic transition. If the time width of the electron pulse (spatial width divided by the speed of an electron) is short compared to the "periods" of motion in the molecule, then, at an intuitive level, the nuclear positions and orientation can be thought of as frozen during the scattering. Whether a pulsed electron beam is short or not depends on the system under investigation. For molecules composed of heavy atoms, the rovibrational periods might be long enough that electron pulses of 1-10 ps width can be considered short whereas 10-100 fs might be needed for molecules with light atoms. When a short electron pulse interacts with a molecule, we expect that the total electronic transition probability will be the convolution of the nuclear position dependence of the transition probability with the probability density of the nuclear wave packet.

Scattering by a pulsed matter beam can be used to control the transitions to specific final states in much the same way that shaped laser pulses can control photochemical reactions. In optical control, the time dependent coupling between a shaped laser pulse and the molecule can be used to guide the reaction path of the molecule. In a very similar manner, a shaped matter beam (for example, a single pulse as in Fig. 1 or a series of pulses etc) can be used to guide the reaction path through the time dependent coupling of the matter beam with the internal (often electronic) degrees of freedom of the molecule; the coherence in a matter beam affects transition probabilities.

The ideas in this paper can be considered to complement those in Refs. ${ }^{9,10}$. In Refs. ${ }^{9,10}$, the diffraction (an essentially elastic scattering process) of a pulsed electron beam from a molecule is used to obtain the time dependent transient structure of molecules. The new feature of this paper is to consider the information that can be obtained if an inelastic scattering occurs.

This paper is organized on two levels. In the first part, we will develop the theory for this system and show how our intuition arises from certain approximations in the calculation of the transition probability. In the second part, we will compare our approximate treatment to a direct numerical solution of the time dependent Schrödinger's equation for a model problem that has all of the features of a real molecule. At the end, we will discuss the limitations of our approximate treatment of 
the scattering and the types of cases where we expect the theory to fail.

In a recent paper $^{2}$, the basic theory was developed for calculating transition probabilities when a quantum target has a non-diagonal density matrix (i.e. in some type of coherent or wave packet state) and the beam also has a non-diagonal density matrix in the direction of the velocity (i.e. the beam has longitudinal coherence). The simplest type of longitudinal coherence (the case treated in this paper) is when the matter beam is a single pulse; this automatically gives non-diagonal terms in the longitudinal density matrix of the beam with momentum difference of roughly Planck's constant divided by the spatial width of the beam. To simplify notation, we will take the electron beam to be traveling in the z-direction; we will assume there is negligible transverse coherence in the beam which introduces other interesting effects that mask the main effect we are investigating. Also, atomic units will be used throughout.

The target is initially in a superposition of several initial states $a$. If the target is completely coherent it can be described as a superposition of energy eigenstates with amplitudes $A_{a}: \Psi=\sum_{a} \psi_{a} A_{a}$. In general the target is only partially coherent and needs to be described with non-diagonal density matrix $\rho_{a a^{\prime}}=\left\langle A_{a} A *_{a^{\prime}}\right\rangle$ at the time of the scattering. The quantum number $a$ specifies the target state completely; at the Born-Oppenheimer level, $a$ would include the electronic state of the molecule, the vibrational level, and the rotational level in the laboratory frame. The transition probability to state $b$ is given by (Eq. (16) of Ref. ${ }^{2}$ )

$$
P_{b} \propto \sum_{a a^{\prime}} \rho_{a a^{\prime}} \xi_{a a^{\prime}}\left(\bar{k}_{z}, \hat{r}\right) \int \rho_{z}(z, z) e^{i \Delta k_{a a^{\prime}} z} d z
$$

where $\bar{k}_{z}$ is the average momentum of the electron beam, $\rho_{z}(z, z)$ is the density of the electron beam in the z-direction at the time of collision, $\Delta k_{a a^{\prime}}=$ $\sqrt{2\left(E_{a}-E_{a^{\prime}}\right)+\bar{k}_{z}^{2}}-\bar{k}_{z} \simeq\left(E_{a}-E_{a^{\prime}}\right) / \bar{k}_{z}$ is the difference in the incident electron's momentum that gives the same final momentum when scattering from states $a$ and $a^{\prime}$, and $\xi_{a a^{\prime}}$ contains scattering information.

The matrix $\xi_{a a^{\prime}}\left(\bar{k}_{z}, \hat{r}\right)$ is a product of scattering amplitudes to go from states $a$ and $a^{\prime}$ and end in state $b$ with the electron scattered into the direction $\hat{r}$ (Eq. (14) of Ref. $^{2}$ ):

$$
\xi_{a a^{\prime}}\left(\bar{k}_{z}, \hat{r}\right) \simeq f_{b \leftarrow a}\left(\bar{k}_{z}, \hat{r}\right) f_{b \leftarrow a^{\prime}}^{*}\left(\bar{k}_{z}, \hat{r}\right)
$$

where $f_{b \leftarrow a}\left(\bar{k}_{z}, \hat{r}\right)$ is the amplitude for the electron to cause the transition from $a$ to $b$ and to scatter into the $\hat{r}$ direction. The diagonal elements of $\xi_{a a}$ are the differential cross sections to scatter from state $a$ into state $b$. The $\simeq$ symbol arises because the change in the final energy of the electron due to the different energies of the initial state $a$ and $a^{\prime}$ has been ignored. Situations where this approximation can fail will be discussed near the end of the paper.
These formulae are somewhat new so the implications may not be clear. Before specializing to molecular systems, we draw attention to some general features. (1) If the density matrix of the target is diagonal $\left(\rho_{a a^{\prime}}=\rho_{a} \delta_{a a^{\prime}}\right.$ with $\rho_{a}$ the probability for being in state $a$ ), then the transition probability reduces to the $\sum_{a} \rho_{a} \xi_{a a}$ which is just the transition probability to state $b$ averaged over the population in the initial states, $a$. (2) If the spatial width of the beam is much larger than $\bar{k}_{z} /\left|E_{a}-E_{a^{\prime}}\right|$ then the Fourier transform in Eq. (1) is essentially zero unless $a=a^{\prime}$; again, the transition probability becomes the weighted average of the transition probability to state $b$. (3) If the scattered electron is not measured, then the transition probability is averaged over all $\hat{r}$ final directions. Unless the scattering from states $a$ and $a^{\prime}$ are in essentially the same final directions, then again the transition probability becomes a weighted average.

Now we will specialize to a molecular case where we expect that the conditions for coherent scattering can be created. For this case, the target molecule is in a rovibrational wave packet state with $\rho_{a a^{\prime}}=A_{a} A_{a^{\prime}}^{*}$. Also, the electron beam is very sharply peaked in space so the Fourier transform does not depend on $\Delta k_{a a^{\prime}}$; this condition means the nuclear positions of the molecule can be treated as frozen during the electron-molecule scattering event. At this level, the transition probability into state $b$ can be written as the square of the absolute value of a transition amplitude:

$$
P_{b} \propto\left|f_{b}\right|^{2}=\left|\sum_{a} f_{b \leftarrow a} A_{a}\right|^{2} .
$$

Actually, this result is not specific to molecules but only depends on the complete coherence of the target and the sharpness of the electron pulse compared to the time scales of the target.

There are several approximations that work particularly well for some molecules. We will use two of these to further the development of the theory. The first is the Born-Oppenheimer approximation and the second is the Franck-Condon principle applied to electronic transitions. For the purposes of this paper, the BornOppenheimer approximation means that the initial state $a$ can be represented as a particular electronic state $i_{a}$ and ro-vibrational state $v_{a}, J_{a}$ to a good approximation (and similarly for the final state). The Franck-Condon principle approximates the transition probability from state $a$ to state $b$ by using the fixed nuclei transition probability between electronic states $f_{i_{b} \leftarrow i_{a}}(\mathbf{R})$ as a function of the nuclear positions $\mathbf{R}$ as a metric in the projection between the initial and final ro-vibrational states:

$$
f_{b \leftarrow a} \simeq \int \chi_{v_{b} J_{b}}^{*}(\mathbf{R}) f_{i_{b} \leftarrow i_{a}}(\mathbf{R}) \chi_{v_{a} J_{a}}(\mathbf{R}) d^{n} \mathbf{R}
$$

where $\chi$ is the wave function for the nuclei.

The Born-Oppenheimer and Franck-Condon approximations can be combined with the general result from Eq. (3) to give a good approximation to the scattering 
amplitude

$$
f_{b} \simeq \int \chi_{v_{b} J_{b}}^{*}(\mathbf{R}) f_{i_{b} \leftarrow i_{a}}(\mathbf{R}) \chi\left(\mathbf{R}, t_{\text {scat }}\right) d^{n} \mathbf{R}
$$

where $\chi\left(\mathbf{R}, t_{\text {scat }}\right)$ is the nuclear wave packet at the time of the scattering. This equation can be thought of as a generalization of the Franck-Condon principle, Eq. (4), to a time dependent initial state. However, it must be remembered that several conditions have been incorporated into this result; the most important is that the time width of the beam is small compared to the shortest rovibrational times of the packet.

An interesting special case is when the experiment does not measure transitions into specific ro-vibrational levels but only measures the transition probability into a specific final electronic state. In this case, the transition probability sums over all of the $v_{b}, J_{b}$ levels. Using completeness of the ro-vibrational levels gives

$$
P_{i_{b}} \propto \int\left|f_{i_{b} \leftarrow i_{a}}(\mathbf{R})\right|^{2}\left|\chi\left(\mathbf{R}, t_{\text {scat }}\right)\right|^{2} d^{n} \mathbf{R}
$$

which is simply the convolution of the electronic transition probability as a function of $\mathbf{R}$ with the initial probability density of the molecular wave packet at the time of the scattering. This is just what is intuitively expected.

Although the theory for pulsed electron scattering from a wave packet target seems fairly simple, it is worthwhile to develop tests in order to ensure that there are no hidden assumptions that will invalidate the derivation. Thus, we have developed a simple model that we can use to test the theory. This model mimics an electron scattering from a vibrating $\mathrm{H}_{2}^{+}$. We have reduced the model to the bare essentials so we could numerically solve Schrödinger's equation to obtain answers without approximation. The model is three-dimensional with direction 3 corresponding to the inter-nuclear distance and directions 1 and 2 corresponding to the two electrons. The Hamiltonian is

$$
H=\frac{p_{1}^{2}}{2}+\frac{p_{2}^{2}}{2}+\frac{p_{3}^{2}}{2 M}+V\left(x_{1}, x_{2}, x_{3}\right)
$$

where the potential is

$$
\begin{aligned}
V & =\frac{1}{\sqrt{1+x_{3}^{2}}}+\frac{1}{\sqrt{1+\left(x_{1}-x_{2}\right)^{2}}} \\
& -\frac{1}{\sqrt{1+\left(x_{1}-x_{3} / 2\right)^{2}}}-\frac{1}{\sqrt{1+\left(x_{1}+x_{3} / 2\right)^{2}}} \\
& -\frac{1}{\sqrt{1+\left(x_{2}-x_{3} / 2\right)^{2}}}-\frac{1}{\sqrt{1+\left(x_{2}+x_{3} / 2\right)^{2}}} .
\end{aligned}
$$

The first two terms in the potential mimic the repulsive Coulomb interaction between the protons and between the electrons. The next two terms give the attractive interaction between the electron 1 and the protons and the last two terms give the attractive interaction between the electron 2 and the protons. $M$ is the reduced mass of the two protons.
The time dependent Schrödinger equation was solved by using the split operator method

$$
\Psi(\delta t) \simeq \exp \left(\frac{-i V \delta t}{2}\right) \exp (-i T \delta t) \exp \left(\frac{-i V \delta t}{2}\right) \Psi(0)
$$

with fast Fourier transforms used to calculate the effect of the kinetic energy operator, $T$. This method has a cumulative error proportional to $\delta t^{2}$ and can be easily programmed to run on massively parallel computers. We performed several runs and reduced the time step until the final result converged. We also varied the number of spatial grid points until convergence was achieved. In the final runs, we used 512 spatial grid points in each direction with the range of the electrons $-100<x_{1}, x_{2}<$ 100 and the range of inter-nuclear distance $0<x_{3}<8$.

As a test, we ran some simple two dimensional calculations to ensure that the approximations in the full derivation were valid. The first test was to check the validity of the Born-Oppenheimer approximation for our model of a vibrating $\mathrm{H}_{2}^{+}$molecular ion. In this test, we solved the one dimensional time dependent radial wave packet on the ground state Born-Oppenheimer potential curve for our model and compared to a full two dimensional solution treating the electron and nuclei simultaneously. The Born-Oppenheimer approximation worked well over one vibrational period as long as the reduced mass of the nuclei was larger than $\sim 10$; the value we used in our simulation was 918. We also used two dimensional time dependent calculations to obtain the fixed nuclei transition probability between the ground electronic state and the first electronically excited state. By monitoring the probability for finding an electron in the first excited state, we could obtain a duration for the electronic collision. This test demonstrated a possible problem for some transitions that will be discussed below.

The calculations of the model quantitatively validated the approximate theory. For the model calculation, the transition probability between the lowest electronic state and the anti-bonding first excited state varied by over a factor of 2 over the range of the nuclear wave packet motion. The approximate and numerical excitation probability agreed to within $5 \%$ over the same range. To give an idea of the accuracy, we plot the probability for one electron to be in the first excited state as a function of inter-nuclear distance, $x_{3}$, at the time of the collision for two cases: Fig. 2 the nuclear wave packet is at the outer turning point and Fig. 3 the nuclear wave packet is at the inner turning point. On the same graphs, we plotted the approximation developed above: $\left|f_{i_{b} \leftarrow i_{a}}(\mathbf{R})\right|^{2}\left|\chi\left(\mathbf{R}, t_{\text {scat }}\right)\right|^{2}$ where $\left|f_{i_{b} \leftarrow i_{a}}\right|^{2}$ was calculated using the frozen nuclei approximation and $\left.\chi\left(\mathbf{R}, t_{\text {scat }}\right)\right|^{2}$ was calculated by time propagating the nuclear wave packet on the Born-Oppenheimer potential surface. We note that even very fine details of the wave packet interference is reproduced. These figures show that the approximations and intuition for pulsed electron scattering from a molecular wave packet are substantially correct.

Several approximations were used to obtain closed 


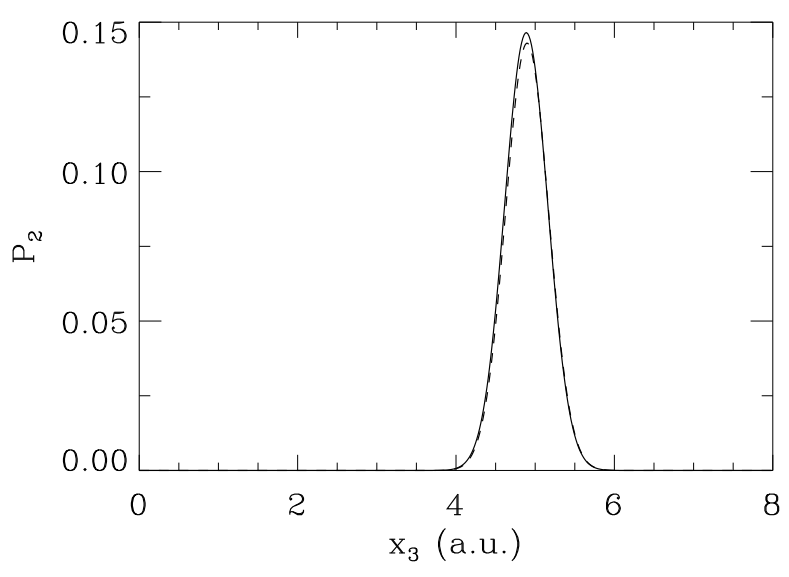

FIG. 2. Dashed line: the product of the Born-Oppenheimer nuclear probability density and the transition probability to the first excited state as a function of inter-nuclear spacing at the time of the collision. Solid line: the probability density for finding one electron in the first excited state as a function of the inter-nuclear spacing from the full 3-dimensional simulation of the scattering.

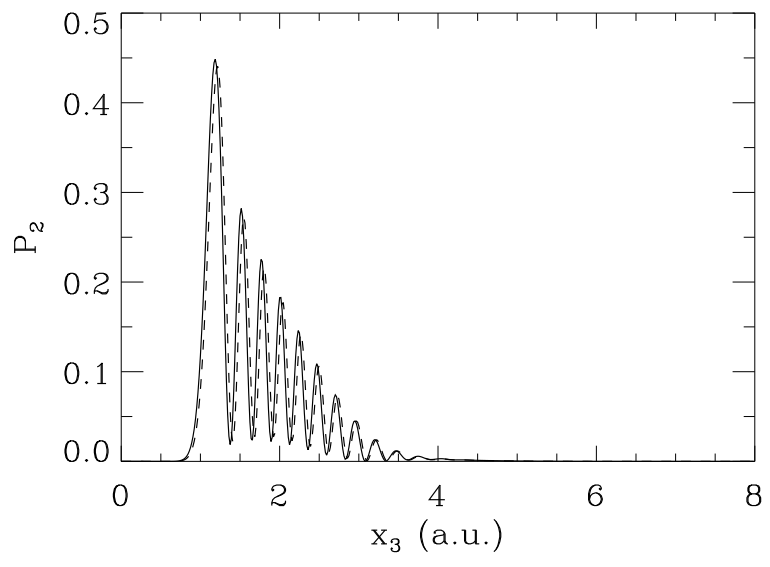

FIG. 3. Same as Fig. 2, but the incident electron wave has been delayed so that the nuclear wave packet is near the inner turning point at the time of the collision. By timing the arrival of the electron pulse, the magnitude of the transition probability to specific final states can be controlled.

form expressions that reproduce our intuition. There are two main approximations that could cause a failure of our treatment. The first is that we have ignored the energy variation of the scattering amplitude over the different eigenstates that make up the initial wave packet. An equivalent way of expressing this approximation is that we have assumed that the duration of the electronmolecule interaction is short compared to the periods of the target; this duration is roughly the distance over which the electron can cause a transition divided by the incident electron's speed. This approximation can fail when the transition is between two electronic levels that have small energy spacing and a dipole coupling; in this case, the electron can cause transitions at large distance and thus the nuclear wave packet can move substantial distances during the collision even when the electron pulse is short. A second approximation is that we have assumed that the scattered electron has a large fraction of its initial energy; i.e., the energy needed to cause a transition is not a large fraction of the incident energy. This is partly related to the first approximation because the duration of the collision can be greatly lengthened if the electron moves slowly after the collision.

In conclusion, we have theoretically investigated the collision of a single electron pulse with a molecular wave packet. We have shown that the electronic transition probability is approximately what is intuitively expected: the transition probability to electronic state $i_{b}$ is the transition probability as a function of the nuclear positions convolved with the nuclear probability density. The approximations were verified by applying the theory to a model system where the fully quantum calculations can be performed nearly exactly. There are two possible uses for pulsed electron scattering from a molecular wave packet. First, the transition probability as a function of the nuclear positions can be experimentally measured by making a peaked nuclear wave packet. If the nuclear wave packet is strongly peaked near the position $\mathbf{R}_{0}$ then the transition probability from the electronic state $i_{a}$ to the final state $i_{b}$ will be proportional to $\left|f_{i_{b} \leftarrow i_{a}}\left(\mathbf{R}_{0}\right)\right|^{2}$. Thus, it would be possible to map out the extent and position of transition states in some cases. This directly implies the second possible use for pulsed electron scattering. As the nuclear wave packet moves on a Born-Oppenheimer potential surface, the transition to different states can change substantially. By timing the arrival of the electron pulse to when the nuclear wave packet reaches a particular region, the transition to specific final electronic states can be enhanced or suppressed. Thus, it should be possible to control the electron-scattering transition into particular electronic states through simple timing techniques.

We acknowledge conversations about this problem with L.D. Noordam. MF was supported by the NSF "Research Experience for Undergraduates" program. FR is supported by the DOE. The calculations were performed at the National Energy Research Center in Berkeley, CA.

1 F. Robicheaux and L.D. Noordam, Phys. Rev. Lett.84,

${ }^{2}$ F. Robicheaux, Phys. Rev. A62 062706 (2000). 3735 (2000). 
${ }^{3}$ F. Robicheaux, G.M. Lankhuijzen, and L.D. Noordam, J. Opt. Soc. Am. B 15, 1-5 (1998).

4 C.W. Rella, et al., J. Opt. Soc. Am. B 16, 182-7 (1999).

5 M.R. Andrews, et al., Science 275, 637 (1997).

6 M.-O. Mewes, et al., Phys. Rev. Lett.78, 582 (1997).

7 I. Bloch, T.W. Haensch, T. Esslinger, Phys. Rev. Lett.82,
3008 (1999).

8 E.W. Hagley, et al., Science 283, 1706 (1999).

9 J. Cao, H. Ihee, and A.H. Zewail, Chem. Phys. Lett. 290, 1 (1998).

10 J.M. Cao, H. Ihee, and A.H. Zewail, P. Natl. Acad. Sci. 96, 338 (1999). 